\title{
What, if Anything, Is an Evolutionary Novelty?
}

\author{
Massimo Pigliucci + t
}

\begin{abstract}
The idea of phenotypic novelty appears throughout the evolutionary literature. Novelties have been defined so broadly as to make the term meaningless and so narrowly as to apply only to a limited number of spectacular structures. Here I examine some of the available definitions of phenotypic novelty and argue that the modern synthesis is ill equipped at explaining novelties. I then discuss three frameworks that may help biologists get a better insight of how novelties arise during evolution but warn that these frameworks should be considered in addition to, and not as potential substitutes of, the modern synthesis.
\end{abstract}

1. Introduction. "Evolutionary novelty" has been a recurring term in the evolutionary biology literature (Nitecki 1990). There is a palpable tension among biologists whenever evolutionary novelties are considered for discussion, with a common fear that the concept will be used as a wedge to undermine the modern synthesis (MS) constituting the currently accepted view of evolutionary theory. Ever since Goldschmidt (1940) professed dissatisfaction with the MS's ability to explain variation across species, his "hopeful monsters" have repeatedly reared their ugly heads and have consistently been beaten back by defenders of the orthodoxy.

Yet, evolutionary novelties persist as a fascinating problem for theorists and experimentalists alike, a problem that has been taken seriously enough to become a major stated goal of a whole field of investigation, known as "evo-devo" (for evolution and/of development). Despite great progress over the past decade at finally incorporating development in the modern

$\dagger$ To contact the author, please write to: Departments of Ecology and Evolution and Philosophy, Stony Brook University, Stony Brook, NY 11794; e-mail: pigliucci@, platofootnote.org.

$\$$ Thanks to Jonathan Kaplan for organizing a PSA symposium on evolutionary novelties, as well as to Jonathan, Alan Love, and Alirio Rosales for critical readings of the manuscript. The National Science Foundation (grant IOB-0450240) supported the development of these ideas.

Philosophy of Science, 75 (December 2008) pp. 887-898. 0031-8248/2008/7505-0033\$10.00

Copyright 2008 by the Philosophy of Science Association. All rights reserved. 
synthesis, however, the uneasiness when the subject of evolutionary novelties is brought up remains. Why?

2. Evolutionary Novelties: Examples versus Concepts. What is an evolutionary novelty? A list of most-often cited examples include the shell of turtles (Cebra-Thomas et al. 2005), flight (Prum 2005), flowers (Albert, Oppenheimer, and Lindqvist 2002), the ability of great tits to open bottles of milk (Kothbauerhellmann 1990), the transition from the jaw to the ear of some bones during the evolution of mammals from reptiles (Brazeau and Ahlberg 2006), eyes (Fernald 2006), hearts (Olson 2006), bipedalism (Richmond and Strait 2000), and the origin of Hox genes (Wagner, Amemiya, and Ruddle 2003).

Depending on who one asks, less spectacular examples of novelties are also cited: new patterns of wing colors in butterflies (Beldade and Brakefield 2002), variations in jaw morphology of cichlid fish (Meyer 1993), or the sexually dimorphic horns of horned beetles (Emlen 1994). It is difficult to see what all of these features have in common, partly because they span all levels of biological organization, from morphological to behavioral to molecular traits. So, is the concept of "evolutionary novelty" a cluster concept (Wittgenstein [1953] 1973, Section 66) that does not admit of precise boundaries, but is nonetheless meaningful, or are we facing a disjointed set of examples whose only loose commonality is that they are interesting to biologists?

One way to approach the question is to examine how biologists have variously defined evolutionary novelties and then to ask which of the commonly cited empirical examples do square with the proposed definitions. Ernst Mayr, a major figure of the MS, defined novelties as "any newly acquired structure or property that permits the performance of a new function, which, in turn, will open a new adaptive zone" (Mayr 1963, 602). This links novelties directly to the ecology of the organism, imposing the stringent criterion that a novelty be connected to the adaptive radiation of a whole group. While some of the empirical examples listed above fit the bill (e.g., bird wings), there are also examples of clear evolutionary novelties - such as the transition from the jaw to the ear of some bones during early mammalian evolution - that are difficult to connect to a radiation (mammals did not radiate until the extinction of the dinosaurs). Moreover, while the various jaws of African cichlids are clearly linked to this group's radiations, it is questionable in what sense variations of a preexisting structure (the jaw) can be considered a "novelty." Indeed, the latter case would in fact fail to meet Mayr's own definition. Major new features can evolve without being causally linked to adaptive radiations, while some features may play a role in radiations despite not being novelties in an interesting sense of the word. 
Next, let us consider Arthur's $(2000,811)$ definition of novelty: "Novelties and apomorphies are essentially the same." Apomorphies are unique derived characters of a taxon, which carry no phylogenetic information. The problem here is that this is so broad that any new character or variation, no matter how trivial, becomes an evolutionary novelty. This is certainly not the kind of puzzle that has made some biologists question the explanatory power of the MS. If Mayr's definition can be set aside because of its coupling of novelties and evolutionary radiations, Arthur's can be discarded as uninformative with respect to the big questions that have fueled the debate about novelties.

The two remaining definitions I wish to consider are best treated together because of their conceptual similarities and the light they shed on what I think is most interesting about novelties. Muller and Wagner (1991, 243) conceptualized a novelty as "a structure that is neither homologous to any structure in the ancestral species nor homonomous to any other structure of the same organism," while West-Eberhard $(2003,198)$ thought of it as "a novel trait [based on] a qualitatively distinct developmental variant." The interesting thing about these two definitions is that they are explicitly developmental in nature (unlike Mayr's), do not refer directly to any specific ecological role of the novelty (again, contra Mayr), and are implicitly evolutionary but not trivially so (unlike Arthur's).

Muller and Wagner's and West-Eberhard's concepts are best understood within the goal of evo-devo: to explicitly include development in the MS (Love 2003). The immediate obstacle that arises once we consider novelties as "qualitatively distinct" from existing traits is that the MS tells us how traits spread in natural populations (by natural selection, drift, etc.) but is silent on how they arise - except in the trivial case of quantitative variants of already existing features.

It is this conceptual distinction that led Muller and Newman (2005) to separate what they call "variation" from "innovation": the problem of variation has been addressed by the MS, while the problem of innovation remains elusive. Analogously, Muller and Newman also separate the concept of novelty from that of adaptation (unlike Mayr), noting that the MS provides us with explanatory tools to understand adaptations (natural selection) but falls short when it comes to the question of novelties.

The second aspect of both Muller and Wagner's and West-Eberhard's definitions is the implied statements they make about evolution. Because of their emphasis on nonhomology, these authors rule out broad conceptions of novelties such as Arthur's. Indeed, as Muller and Newman (2005) point out, while discussing Arthur (2000), "some apomorphies are rooted in novelties, others are not," so that the concept of apomorphy is entirely perpendicular to that of evolutionary novelty.

Yet, there are remaining issues even with Muller and Wagner's and 
West-Eberhard's conceptualizations. On the one hand, they do not include any reference at all to ecology, turning the problem almost exclusively into one pertaining to developmental biology. Second, they may err on the side of stressing discontinuity too much, ignoring conceptualizations of evolution as using previously existing structures in novel ways, what Jacob (1977) famously referred to as "tinkering."

I do not put a high premium on precise definitions; indeed, I think that evolutionary novelties are a necessarily fuzzy concept, like that of biological species (Pigliucci 2003). Nonetheless, it seems to me that these two deficiencies need to be addressed, which is why I venture to propose an amended definition of evolutionary novelties: Evolutionary novelties are new traits or behaviors, or novel combinations of previously existing traits or behaviors, arising during the evolution of a lineage, and that perform a new function within the ecology of that lineage. This definition does not imply any specific mechanism or long-term evolutionary effect, since the first one is precisely the objective of empirical research in this area and the second one is a matter to be settled based on the historical record. However, my definition (1) makes explicit the fact that often novelties are not absolute discontinuities but can be built on previously existing parts, (2) indicates that they are a phenomenon that affects the evolution of certain lineages without implying that all derived characters are in fact novelties, and (3) requires some kind of ecological function to eventually be coupled with the novelty, although it does not imply a necessary link between novelties and adaptive radiations.

3. Why Is the Modern Synthesis Insufficient? "Describing a single individual is as theoretically peripheral to a populationist as describing the motion of a single molecule is to the kinetic theory of gases. In this important sense, population thinking involves ignoring individuals" (Sober 1980, 370). As mentioned above, several authors have hinted at the fact that the modern synthesis is not sufficient to account for evolutionary novelties, implying more or less forcefully that the MS is incomplete in some major respect (Schlichting and Pigliucci 1998; Gould 2002). This has in turn led to unnecessarily defensive reactions by supporters of the MS, who apparently often confuse the possibility of an expansion of the MS with an outright rejection of it.

The MS arose out of a necessity to reconcile classic Darwinian ideas and the new fields of Mendelian and statistical genetics (Mayr and Provine 1980). It achieved an account of how variation and heredity come about and effectively unified natural history, Mendelian genetics, population biology, and paleontology. Famously, what was left out of the synthesis was development, despite the large amount of work on the embryology of both plants and animals that had been done up to that point. Equally 
famously, the synthesis did not address what some biologists perceived as a different kind of phenotypic variation, the one differentiating species and higher taxa and, in particular, what we now refer to as evolutionary novelties. This culminated in Goldschmidt's (1940) bold attempt at expansion (again, not rejection) of the MS, an attempt that led to vehement dismissal by the synthesists, especially Mayr.

It is this conceptual history that allows us to make sense of the enthusiasm that has greeted the onset of evo-devo in the 1990s: on the one hand, here finally was a chance to put development back into the MS, made possible by the advances in understanding and the technical possibilities of molecular biology; on the other hand, perhaps we were finally going to come up with a reasonable explanation for the hidden problem of innovation and definitively put to rest the specter of hopeful monsters.

It is still early to say what evo-devo will or will not be able to accomplish, but some things are clear enough to illuminate this discussion. First, much evo-devo research so far is more about "devo" than about "evo" and has little at all to do with ecology (in turn, a strangely unnoticed additional missing piece from the MS). Therefore, despite some spectacular discoveries (such as the evolutionary conservation of Hox genes [Lemon and McGinnis 2006]), there is little reason to expect this line of inquiry to offer a fresh way of looking at the problem of novelties. Second, to the extent that evo-devo has expressly attempted to link up with the MS and its population-level approach, this has been through the study of the molecular-developmental underpinnings of quantitative variation, which means that evo-devo still addresses primarily the problem that has already been dealt with by the MS (Pigliucci and Kaplan 2006). To put it simply, the question is not, say, how different species of turtles get different colors or shapes of their shells; the problem is how do we account for the transition between reptiles without and with shells in the first place?

4. What Other Explanatory Frameworks? Well, then, are there any additional (not "alternative") explanatory principles the use of which biologists may profitably explore to extend the modern synthesis and account for evolutionary novelties? There are at least three promising candidates, each of which has received some attention over the years and that may need to be combined to some extent in order to satisfactorily expand the synthesis: complexity theory, phenotypic/genetic accommodation, and epigenetic inheritance systems.

4.1. Complexity Theory. Complexity theory is a vast and heterogeneous field of mathematical research (Bak and Chen 1991). It has been explicitly applied to evolutionary theory (Sneppen et al. 1995) and even to the 
specific question of evolutionary novelties (Toquenaga and Wade 1996). The idea is that certain kinds of systems, those characterized by an intermediate level of connectivity among their components (like a limited degree of epistasis in genetic networks), exhibit surprisingly complex behaviors that are emergent properties of the interactions among the system's components.

Some complex systems exhibit a number of stable states between which the system rapidly transitions, spending little time in intermediate states. These "attractors" open up the tantalizing possibility that shifts in phenotype (say, from nonshelled to shelled reptiles) may occur rapidly because the beginning and ending states are stable islands in phenotypic space, while the intermediate forms are not. In this sense, biological systems may share formal commonalities with a large number of natural (e.g., hurricanes) and artificial (e.g., economies) systems that would make the evolution of phenotypes in biology partly (although not entirely) a matter of generalizable laws of form, a project that goes back at least to D'Arcy Thompson ([1917] 1942).

The problem with complexity theory, at least as perceived by most biologists, resides precisely in its focus on the formal similarities among different kinds of systems, so much so that it is often regarded as little more than a metaphor. Equally important, and related to the same issue, is the fact that complexity theory seems to be able to come up "only" with qualitative descriptions, significantly deviating from the ideal of quantitative mathematics established in physics and consciously imported into the MS by people like Fisher, and which eventually gave us the statistical treatments of evolutionary quantitative genetics.

This sense of apparent limitation of complexity theory may have resulted in its approach having already peaked in biology during the 1990s, a fate similar to a predecessor of the theory that similarly promised a mathematical treatment of qualitative phenomena: Thom's (1977) "catastrophe theory." The latter also enjoyed a brief period of application in biology and was invoked by Waddington (1979) as a way to expand the MS. Thom's attempt suffered from the fact that, while the math allowed for enticing graphical descriptions of sudden state transitions, it was so general that it was difficult to go beyond its use as a metaphor in any specific scientific context. Then again, recently Gavrilets (1999) has remarked that in evolutionary biology models should be thought of as playing the part of useful metaphors, rather than of providing specific predictions.

There is some recent evidence that the idea of phenotypic attractors may as yet generate empirically parametrized models that are close enough to "real" biology to be palatable to biologists. For example, work by Salazar-Ciudad and Jernvall (2005) on the genotype-phenotype mapping 
of teeth morphology in mammals indicates that while simple phenotypes follow the pattern expected by the MS (small genetic changes correspond to small phenotypic changes), complex phenotypes are characterized by rapid evolutionary transitions without intermediate forms.

4.2. Phenotypic and Genetic Accommodation. Phenotypic and genetic accommodation are phenomena highlighted by West-Eberhard (2003) that relate to Waddington's idea of genetic assimilation (Pigliucci and Murren 2003) and to the concept of phenotypic plasticity (Pigliucci 2001). Phenotypic accommodation is a well-documented phenomenon by which the inherent plasticity of developmental systems extends to the changes in the environment internal to the organism, triggering a concerted series of accommodations. A classic - and surprisingly understudied - example is the spontaneous appearance of bipedalism in a variety of vertebrates (West-Eberhard 2003). The initial impetus may be due to a mutation or an environmental accident (e.g., a viral infection) that impairs the use of the forelimbs in the newborn. Subsequently, the developmental trajectory of bones, cartilage, muscles, and tendons is altered to plastically "accommodate" the new situation. The adult animal displays a host of complex morphological alterations that, if found in the fossil record, would "clearly" point to a long process of "mosaic" evolution (the MS explanatory principle for such cases). Moreover, the behavior of the animal is also altered and may indeed channel said morphological changes.

But how can this sort of accident be of evolutionary importance? It can because the change can eventually become fixed by genetic mutations, if the new phenotype is advantageous and/or if the conditions that generated it (e.g., an environmental or behavioral change) are persistent enough to allow the standard process of mutation and natural selection to "assimilate" the novelty. Again, it is remarkable, in this context, that very little serious attention has been paid to the possible origin of bipedalism through behaviorally or environmentally induced phenotypic accommodation.

The key point of phenotypic-genetic accommodation is that the genetic change follows, rather than precedes, the phenotypic change. Moreover, because the initial stimulus is environmental or behavioral, it can simultaneously affect several individuals in a given population, which bypasses another infamous problem faced by the standard MS account: even beneficial genetic changes are initially at low frequency, and they are predicted to often disappear from the population via drift before becoming common enough that natural selection can fix them.

4.3. Epigenetic Inheritance Systems. Epigenetic inheritance systems (EIS) comprise a broad category of phenomena that have been under 
recent intense study in molecular developmental biology and are beginning to be taken seriously by evolutionary biologists (Jablonka and Lamb 2005). The basic idea is that there is more than genetics to the study of inheritance, as behaviors, cultural attributes, and nongenetic modifications of gene expression (e.g., via DNA methylation) can all be passed to the next generation without any change in DNA sequence. In a sense, EIS is an example of scientific application of the broader philosophical framework known as developmental systems theory (Oyama, Griffiths, and Gray 2001), the idea that inheritance comprises the whole set of developmental resources, including genes, epigenetic effects, and environmental resources necessary to recreate an organismal system.

There are several molecular mechanisms that make epigenetic inheritance possible, including histone modification, small interfering RNAs, and DNA methylation patterns, though the latter are by far the best understood. For example, methyl groups attached to certain regions of the DNA strand of a chromosome alter the expression of the genes comprised in those regions of DNA. Sometimes these patterns of methylation are reset in the germ line, and their modifications are therefore not heritable, but we now know of cases in both plants and animals were there is no reset, or the reset is only partial. Moreover, epigenetic systems can be affected by environmental signals, such as a variety of stresses. This, one should hasten to say, does not have anything to do with Lamackiantype inheritance of "acquired" characteristics, because the environmentally induced changes are still random with respect to adaptation.

What makes EIS relevant to evolutionary theory is that they may represent a reservoir of heritable variation above and beyond the classic one residing in DNA sequences. Furthermore, EIS have been implicated in macroevolutionary phenomena such as interspecific hybridization and polyploidy (Salmon, Ainouche, and Wendel 2005). Even more fascinatingly, EIS interact in complex fashion with standard genetic inheritance, not only because EIS alter gene expression but because genes themselves encode the proteins necessary for the very existence of EIS. The combinatorial possibilities are mind-boggling, and it seems likely that some of this hitherto unexpected variation may help us understand the problem of innovation that underlies the evolution of phenotypic novelties.

5. Conclusion: Where To Next? Popper famously wrote that "[Darwinian theory] never gives us a full explanation of anything's coming into being in the course of evolution," because it is a theory that assumes variation and "therefore . . . cannot really explain it. . . . It is strictly a theory of genes, yet the phenomenon that has to be explained in evolution is that of the transmutation of form" (Popper and Eccles 1977, 560, note 1, in Platnick and Rosen 1987, 13). It is because the modern synthesis is a 
theory of genes, not of phenotypes, that it is incomplete. Of course, being incomplete is not the same as being wrong, which is why I argue-with Gould (2002) - that we are still building on the foundations laid out by Darwin almost 150 years ago and that no earth-shattering paradigm shift is in the works. Yet, "defenders" of the synthesis have been busy over the past several decades denying that we need anything more than the conceptual tools that the MS already affords us (Stebbins and Ayala 1981).

Let me put it clearly: while the MS has been very successful at expanding Darwinism to account for genetics and population biology, it has failed to sensibly incorporate both developmental biology and ecology; while the MS has given us an account of genetic variation and of how it changes in populations over time, it has reached an impasse on the question of the origin and evolution of phenotypic novelties and organismal body plans. Since the MS has had several decades to try, it seems sensible to seriously explore some of the potential additions and see where they might lead us.

I have outlined three promising venues of theoretical and empirical research that have the potential of providing new insights into the question of evolutionary novelties. These may or may not pan out, of course, but it seems that they - especially in combination - provide evolutionary biologists with enough food for thought to be taken seriously and to force us to go beyond the standard hand-waving argument of MS supporters that "this is already adequately explained by the synthesis."

From a practical standpoint, I suggest that biologists concentrate on a limited number of "model systems" to understand phenotypic novelties. The use of model systems in biology has a long, and sometimes controversial, history. Fruit flies, rats, and Arabidopsis plants have been adopted as model systems in genetics, behavioral biology, and developmental biology, respectively. The success of this approach is based on the idea that by concentrating efforts on a small number of organisms one can learn much more about them than by spreading efforts on a larger, albeit more representative, group. As an example, I put together Table 1 to list a number of potential model systems for the understanding of evolutionary novelties.

It seems to me that to understand an evolutionary novelty, by and large, means to be able to provide an account of four components, listed in the Table: its history (in terms of fossil record or in the sense of sequence of events on independently reconstructed phylogenies); why it came to be frequent in a population (i.e., its functional ecology); how is it made possible at the developmental level; and what sort of genetic underpinning helps instantiate it. Notice from the Table that it is hard to find systems where all these questions have been answered to a reasonable degree of 
TABle 1. Examples of Model Systems That Can Be Used to Study EVOLUTIONARY NOVELTIES.

\begin{tabular}{|c|c|c|c|c|}
\hline System & $\begin{array}{c}\text { Fossil/ } \\
\text { Phylogenetic } \\
\text { Record? } \\
\text { (History) }\end{array}$ & $\begin{array}{c}\text { Functional } \\
\text { Ecology? } \\
\text { (Why?) }\end{array}$ & $\begin{array}{c}\text { Developmental } \\
\text { Biology? } \\
\text { (How?) }\end{array}$ & $\begin{array}{c}\text { Molecular } \\
\text { Biology? (How?) }\end{array}$ \\
\hline Turtle shell & No & Some & Some & Little \\
\hline Bird wings and feathers & Little & Some & Yes & Some \\
\hline Vertebrate eye & No & Yes & Yes & Some \\
\hline Mandible-ear transition & Yes & Some & Yes & Little \\
\hline Heart & Yes & Yes & Some & Some \\
\hline $\begin{array}{l}\text { Milk-opening behavior } \\
\text { (in great tits) }\end{array}$ & N/A & Yes & N/A & No \\
\hline Flowers & Little & Some & Yes & Yes \\
\hline Hox genes & N/A & Some & N/A & Yes \\
\hline
\end{tabular}

satisfaction, but the point is that this is the sort of thing we would like to know in order to understand evolutionary novelties.

From a theoretical and philosophical standpoint, what we need to articulate is how precisely the additional conceptual elements I have briefly sketched above (as well as possibly others, such as niche construction; Odling-Smee et al. 2003) exactly fit with each other and with the already existing MS framework. This is a research program that obviously is beyond the scope of this essay but that nevertheless seems ripe for pursuit and should unfold over the foreseeable future.

\section{REFERENCES}

Albert, V. A., D. G. Oppenheimer, and C. Lindqvist (2002), "Pleiotropy, Redundancy and the Evolution of Flowers", Trends in Plant Science 7: 297-301.

Arthur, W. (2000), "Intraspecific Variation in Developmental Characters: The Origin of Evolutionary Novelties", American Zoologist 40: 811-818.

Bak, P., and K. Chen (1991), "Self-Organized Criticality", Scientific American (January): 46-53.

Beldade, P., and P. M. Brakefield (2002), "The Genetics and Evo-Devo of Butterfly Wing Patterns", Nature Genetics 3: 442-452.

Brazeau, M. D., and P. E. Ahlberg (2006), "Tetrapod-like Middle Ear Architecture in a Devonian Fish", Nature 439 (7074): 318-321.

Cebra-Thomas, J., F. Tan, S. Sistla, E. Estes, G. Bender, C. Kim, P. Riccio, and S. F. Gilbert (2005), "How the Turtle Forms Its Shell: A Paracrine Hypothesis of Carapace Formation", Journal of Experimental Zoology 304B: 558-569.

Emlen, S. T. (1994), "Benefits, Constraints and the Evolution of the Family", Trends in Ecology and Evolution 9: 282-284.

Fernald, R. D. (2006), "Casting a Genetic Light on the Evolution of Eyes", Science 313: 1914-1918.

Gavrilets, S. (1999), “A Dynamical Theory of Speciation on Holey Adaptive Landscapes”, American Naturalist 154: 1-22.

Goldschmidt, R. (1940), The Material Basis of Evolution. New Haven, CT: Yale University Press.

Gould, S. J. (2002), The Structure of Evolutionary Theory. Cambridge, MA: Harvard University Press. 
Jablonka, E., and M. J. Lamb (2005), Evolution in Four Dimensions: Genetic, Epigenetic, Behavioral, and Symbolic Variation in the History of Life. Cambridge, MA: MIT Press. Jacob, F. (1977), "Evolution and Tinkering", Science 196: 1161-1166.

Kothbauerhellmann, R. (1990), "On the Origin of a Tradition-Milk Bottle Opening by Titmice (Aves, Paridae)", Zoologischer Anzeiger 225 (5-6): 353-361.

Lemons, D., and W. McGinnis (2006), "Genomic Evolution of Hox Gene Clusters", Science 313: $1918-1922$.

Love, A. C. (2003), "Evolutionary Morphology, Innovation, and the Synthesis of Evolutionary and Developmental Biology", Biology and Philosophy 18: 309-345.

Mayr, E. (1963), Animal Species and Evolution. Cambridge, MA: Harvard University Press.

Mayr, E., and W. B. Provine (1980), The Evolutionary Synthesis: Perspectives on the Unification of Biology. Cambridge, MA: Harvard University Press.

Meyer, A. (1993), "Phylogenetic Relationships and Evolutionary Processes in East African Cichlid Fishes", Trends in Ecology and Evolution 8: 279-284.

Muller, G. B., and S. A. Newman (2005), "The Innovation Triad: An EvoDevo Agenda", Journal of Experimental Zoology 304B: 487-503.

Muller, G. B., and G. P. Wagner (1991), "Novelty in Evolution: Restructuring the Concept", Annual Review of Ecology and Systematics 22: 229-256.

Nitecki, M. H., ed. (1990), Evolutionary Innovations. Chicago: University of Chicago Press.

Odling-Smee, J. F., K. N. Laland, and M. W. Feldman (2003), Niche Construction: The Neglected Process in Evolution. Princeton, NJ: Princeton University Press.

Olson, E. N. (2006), "Gene Regulatory Networks in the Evolution and Development of the Heart", Science 313: 1922-1927.

Oyama, S., P. E. Griffiths, and R. D. Gray, eds. (2001), Cycles of Contingency: Developmental Systems Theory and Evolution. Cambridge, MA: MIT Press.

Pigliucci, M. (2001), Phenotypic Plasticity: Beyond Nature and Nurture. Baltimore: Johns Hopkins University Press.

(2003), "Species as Family Resemblance Concepts: The (Dis-)Solution of the Species Problem?" BioEssays 25: 596-602.

Pigliucci, M., and J. Kaplan (2006), Making Sense of Evolution: Toward a Coherent Picture of Evolutionary Theory. Chicago: University of Chicago Press.

Pigliucci, M., and C. Murren (2003), "Genetic Assimilation and a Possible Evolutionary Paradox: Can Macroevolution Sometimes Be So Fast as to Pass Us By?", Evolution 57: 1455-1464.

Platnick, N. I., and D. E. Rosen (1987), "Popper and Evolutionary Novelties", History and Philosophy of Life Sciences 9: 5-16.

Popper, K. R., and J. C. Eccles (1977), The Self and Its Brain: An Argument for Interactionism. Berlin: Springer.

Prum, R. O. (2005), "Evolution of the Morphological Innovations of Feathers", Journal of Experimental Zoology 304B: 570-579.

Richmond, B. G., and D. S. Strait (2000), "Evidence That Humans Evolved from a KnuckleWalking Ancestor", Nature 404: 382-385.

Salazar-Ciudad, I., and J. Jernvall (2005), "Graduality and Innovation in the Evolution of Complex Phenotypes: Insights from Development", Journal of Experimental Zoology 304B: 619-631.

Salmon, A., M. L. Ainouche, and J. F. Wendel (2005), "Genetic and Epigenetic Consequences of Recent Hybridization and Polyploidy in Spartina (Poaceae)", Molecular Ecology 14: $1163-1175$.

Schlichting, C. D., and M. Pigliucci (1998), Phenotypic Evolution: A Reaction Norm Perspective. Sunderland, MA: Sinauer.

Sneppen, K., P. Bak, H. Flyvbjerg, and M. H. Jensen (1995), "Evolution as a Self-Organized Critical Phenomenon", Proceedings of the National Academy of Science USA 92: 52095213.

Sober, E. (1980), "Evolution, Population Thinking, and Essentialism", Philosophy of Science 47: $350-383$.

Stebbins, G. L., and F. J. Ayala (1981), "Is a New Evolutionary Synthesis Necessary?", Science 213: 967-971. 
Thom, R. (1977), "Catastrophe Theory", Nature 270 (5639): 658.

Thompson, D. ([1917] 1942), On Growth and Form. Cambridge: Cambridge University Press.

Toquenaga, Y., and M. J. Wade (1996), "Sewall Wright Meets Artificial Life: The Origin and Maintenance of Evolutionary Novelty", Trends in Ecology and Evolution 11: 478482.

Waddington, C. H. (1979), "A Catastrophe Theory of Evolution”, Annals of the New York Academy of Sciences 231: 32-42.

Wagner, G. P., C. Amemiya, and F. Ruddle (2003), "Hox Cluster Duplications and the Opportunity for Evolutionary Novelties", Proceedings of the National Academy of Sciences USA 100: 14606-14606.

West-Eberhard, M. J. (2003), Developmental Plasticity and Evolution. Oxford: Oxford University Press.

Wittgenstein, L. ([1953] 1973), Philosophical Investigations. New York: Macmillan. 